\title{
Distinct brain structure and behavior related to ADHD and conduct disorder traits
}

\author{
Frida Bayard ${ }^{1}$. Charlotte Nymberg Thunell ${ }^{1}$ Christoph Abé ${ }^{1} \cdot$ Rita Almeida $^{2} \cdot$ Tobias Banaschewski $^{3}$. \\ Gareth Barker $\mathbb{D}^{4} \cdot$ Arun L. W. Bokde ${ }^{5}$ Uli Bromberg ${ }^{6}$. Christian Büchel ${ }^{6}$. Erin Burke Quinlan ${ }^{7}$. \\ Sylvane Desrivières $\mathbb{1}^{7} \cdot$ Herta Flor ${ }^{8,9} \cdot$ Vincent Frouin $^{10}{ }^{10} \cdot$ Hugh Garavan $^{11} \cdot$ Penny Gowland $^{12} \cdot$ Andreas Heinz $^{13}$. \\ Bernd Ittermann ${ }^{14}$ - Jean-Luc Martinot ${ }^{15}$ - Marie-Laure Paillère Martinot ${ }^{16}$ • Frauke Nees $^{3,8}$. \\ Dimitri Papadopoulos Orfanos $\mathbb{D}^{10} \cdot$ Tomáś Paus $^{17} \cdot$ Luise Poustka $^{18,19} \cdot$ Patricia Conrod $^{20} \cdot$ Argyris Stringaris $^{21}$. \\ Maren Struve $^{3}$ - Jani Penttilä ${ }^{22} \cdot$ Viola Kappel $^{23} \cdot$ Yvonne Grimmer $^{3} \cdot$ Tahmine Fadai $^{6}$ - Betteke van Noort ${ }^{24}$. \\ Michael N. Smolka $\mathbb{C}^{25} \cdot$ Nora C. Vetter ${ }^{25} \cdot$ Henrik Walter $\mathbb{D}^{13} \cdot$ Robert Whelan $^{26} \cdot$ Gunter Schumann $^{7}$. \\ Predrag Petrovic ${ }^{1} \cdot$ the IMAGEN Consortium
}

Received: 16 April 2017 / Revised: 22 March 2018 / Accepted: 5 April 2018 / Published online: 14 August 2018

(C) Springer Nature Limited 2018

\begin{abstract}
Attention-Deficit/Hyperactivity Disorder (ADHD) and conduct disorder (CD) exemplify top-down dysregulation conditions that show a large comorbidity and shared genetics. At the same time, they entail two different types of symptomology involving mainly non-emotional or emotional dysregulation. Few studies have tried to separate the specific biology underlying these two dimensions. It has also been suggested that both types of conditions consist of extreme cases in the general population where the symptoms are widely distributed. Here we test whether brain structure is specifically associated to ADHD or CD symptoms in a general population of adolescents $(n=1093)$ being part of the IMAGEN project. Both ADHD symptoms and CD symptoms were related to similar and overlapping MRI findings of a smaller structure in prefrontal and anterior cingulate cortex. However, our regions of interest (ROI) approach indicated that gray matter volume (GMV) and surface area (SA) in dorsolateral/dorsomedial prefrontal cortex and caudal anterior cingulate cortex were negatively associated to ADHD symptoms when controlling for CD symptoms while rostral anterior cingulate cortex GMV was negatively associated to CD symptoms when controlling for ADHD symptoms. The structural findings were mirrored in performance of neuropsychological tests dependent on prefrontal and anterior cingulate regions, showing that while performance on the Stop Signal test was specifically related to the ADHD trait, delayed discounting and working memory were related to both ADHD and CD traits. These results point towards a partially domain specific and dimensional capacity in different top-down regulatory systems associated with ADHD and CD symptoms.
\end{abstract}

\section{Introduction}

Top-down dysregulation of information processing is a core mechanism underlying many psychiatric disorders. For

These authors contributed equally: Frida Bayard, Charlotte Nymberg.

Electronic supplementary material The online version of this article (https://doi.org/10.1038/s41380-018-0202-6) contains supplementary material, which is available to authorized users.

Predrag Petrovic

predrag.petrovic@ki.se

Extended author information available on the last page of the article. example, in Attention-Deficit/Hyperactivity Disorder (ADHD)-defined mainly by non-emotional symptoms including impulsivity, hyperactivity, and inattention-a core theory has evolved around deficits in executive functions (EF) $[1,2]$. EF consists of a set of cognitive control processes associated with top-down regulatory systems in the brain $[1,2]$. In line with this hypothesis, reported ADHDsymptoms have been associated with poor results in EF tests although the relation has been surprisingly weak and unspecific [3].

In addition to ADHD, there are also a set of psychiatric disorders which are characterized by top-down dysregulation in emotional processing. These include Borderline Personality Disorder (BPD) and Antisocial Personality 
Disorder (ASPD) in adults, and Conduct Disorder (CD) in children and adolescents [2, 4-7]. They all comprise emotional instability symptoms (partially overlapping with the concept of emotional lability) such as rapidly shifting emotional states and affective behavior. Also, altered emotional processing and regulation have been noted in these states [2]. Collectively, these disorders may thus serve as valuable models for studying emotional dysregulation. ADHD and emotional instability disorders show substantial symptom overlap and are highly comorbid [8-11]. A shared genetic contribution [12] has also been suggested. The overlapping relation between ADHD and emotional instability disorders has made it problematic to isolate behaviors and mechanisms specific to each condition.

A model encompassing both ADHD and emotional instability disorders has recently been proposed [2]. The model builds conceptually on the research domain criteria (RDoC) approach [2,13] and suggests that both forms of disorders include similar top-down dysregulation-with the difference being whether non-emotional or emotional regulatory processes are predominantly affected. An important aspect of the model is that both non-emotional and emotional regulatory capacities are likely to be dimensional and vary among individuals even on a subclinical level within a healthy population [14-16]. In addition, some forms of ADHD may show a strong degree of subclinical emotional dysregulation in the absence of other comorbidities [2, 17-19].

In children and adolescents, a clinical relation between ADHD and CD has been noted [6]. Although CD involves antisocial behaviors, which are not observed in pure ADHD, both disorders share common symptomatology including problems with sustained attention, cognitive switching, and inhibition [6]. The fact that these two disorders are highly comorbid [11] has often not been considered and might have confounded the findings of previous studies. Thus, it is still not entirely clear which of the features are specific to each disorder. Some studies have compared patients with pure ADHD and CD (without comorbidities), proposing the presence of a few disorderspecific behaviors and brain functional characteristics [6]. However, it has been suggested that the degree of ADHD symptoms is related to the degree of CD symptoms even on a sub-clinical level [2]. It is thus possible that studies on ADHD still may be confounded by $\mathrm{CD}$ features and vice versa. It is therefore of importance to consider both symptom dimensions simultaneously even on a sub-clinical level in relation to behavioral and neurobiological features. An effective approach to better understand the specific behavioral and neurobiological features in these two dimensions is to study the different traits in community populations.

Impaired performance on several $\mathrm{EF}$ tests in ADHD [20-22] are associated with deficits in top-down regulatory systems of non-emotional processes [2, 17, 18, 23]. Especially EF tests that measure inhibition, switching, setshifting, updating, and sustained attention (including Stop Signal test, Stroop test, Wisconsin Card Sorting Test and Continuous Performance Test) have been shown to be altered in ADHD [3]. Further, working memory (WM), which partially consists of several EF components, has shown to be significantly affected in ADHD [3]. Functional imaging studies have consistently revealed networks underlying non-emotional or "cool" EF processes involving dorsolateral and dorsomedial prefrontal cortex (dl/dmPFC), ventrolateral PFC (vlPFC), and caudal anterior cingulate cortex (cACC), which work in conjunction with parietal and subcortical regions such as the striatum [24, 25]. These networks interact with the dopamine system and other specific neuromodulatory systems [2]. The aforementioned brain regions (including $\mathrm{dl} / \mathrm{dmPFC}$, vlPFC, and $\mathrm{cACC}$ ) are known to be less activated during $\mathrm{EF}$ tasks in ADHD patients vs. controls [26-29]. On a brain structure level it has been shown that the "ADHD-brain" matures significantly slower than for healthy individuals-especially in the prefrontal and anterior cingulate areas-with an delay of approximately three years (when medians for the two groups were comapred) [30]. Although some of these structural differences seem to disappear in adulthood [31], some studies indicate smaller $\mathrm{dl} / \mathrm{dmPFC}$ and $\mathrm{cACC}$ volume also in adults with ADHD [32]. Recent meta-analyses [29, 33, 34] have also shown decreased gray matter volume (GMV) in insula, ventromedial prefrontal/medial orbitofrontal cortex, rostral anterior cingulate cortex (rACC), and occipital lobe and deep structures such as amygdala, basal ganglia (including nucleus accumbens), and hippocampus in individuals with ADHD. A major drawback in most previous brain imaging studies on ADHD (including the above mentioned meta-analyses) is that they have often not controlled for other sub-clinical symptoms such as traits of CD.

In analogy with ADHD, it has been suggested that emotional instability disorders show a lack of regulatory capacity for emotional processes including emotional aspects of EF, i.e., "hot" EF [2, 4-7]. Although there is a large overlap between emotional and non-emotional regulatory processes, there seems to be some specificity, and it has been suggested that lateral orbitofrontal cortex (1OFC) and rACC are more involved in the emotional subcomponents of top-down regulation [2]. In line with this, it has been observed that these brain regions are less activated in emotional regulation and "hot" EF tasks in emotional instability disorders as compared to controls [35-41]. Moreover, several well-powered studies have indicated a smaller volume in 1OFC and rACC in patients with these disorders [42-44]. Specifically for CD, recent large singlesite studies and meta-analyses suggest a smaller GMV in a 
number of prefrontal structures including 1OFC, ventrolateral PFC (vlPFC), medial prefrontal extending into rACC but also other structures involved in emotional processing such as insula and amygdala [45, 46]. However, these studies have rarely controlled for non-emotional dysregulation or ADHD symptoms.

In the present study, we analyzed data from a large sample of adolescents $(N=1093)$ from the IMAGEN project [47]. While the general relationship between brain structure and ADHD traits has been presented in a recent VBM analysis [48], here we aim to specifically disentangle effects of ADHD and CD traits on both brain structure and behavioral domains. We hypothesized that both ADHD and $\mathrm{CD}$ traits are inversely related to common overlapping brain areas including PFC and ACC and in addition will show specific structural signatures unique to the traits. We expected: (1) that ADHD trait will be negatively related to cortical volume in regions involved in non-emotional topdown regulation and cool EF (e.g., dl/dmPFC and cACC) even when controlling for $\mathrm{CD}$ trait. (2) Negative correlations between $\mathrm{CD}$ trait and cortical volume in regions involved in emotional regulation (e.g., lOFC and rACC) even after correcting for ADHD trait. We primarily used a region of interest (ROI) approach in order to increase sensitivity (including ROIs of $\mathrm{dl} / \mathrm{dmPFC}$, cACC $1 \mathrm{OFC}$, and rACC). Other prefrontal areas, such as vlPFC, have a less clear specificity, as they seem to be equally involved in emotional and non-emotional regulatory processes $[24,25,49,50]$. The hypothesis and analysis plan were preregistered within IMAGEN before access to the data was granted. Although our hypothesis is based on cortical volume measures, surface-based methods provided by FreeSurfer allowed the exploration of cortical thickness and surface area measures. We also tested whether a similar division between the traits was mirrored in behavioral tests of EF and delay discounting.

\section{Materials and methods}

\section{Participants}

Data was obtained from a community sample consisting of 1969 adolescents from the European multi-center geneticneuroimaging IMAGEN study (https://imagen-europe.com) [47]. Ethical approval had been obtained by the local ethics committees and written informed consent from all participants and their legal guardians. Recruitment procedures have been described in a previous review [47]. Participants completed neuropsychological, clinical, and personality assessments online and at assessments sites. A total of $N=1093$ participants (mean age: $14.47 \pm 0.39$ years; 616 females) provided sufficient MRI data quality and were analyzed in this study (Supplementary Fig. 1). Sample characteristics are described in Supplementary Table 1.

\section{ADHD and CD traits}

ADHD and CD symptoms were measured using the Strength and Difficulties Questionnaire (SDQ) [51]. Its psychometric properties and reliability have been described by Goodman et al. [51]. SDQ consists of five subscales; Emotional problems scale, Conduct problems scale, Hyperactivity/Inattention scale, Peer problems scale, and Prosocial scale. While the Conduct problems scale has been constructed to measure $\mathrm{CD}$ symptoms and mirrors the categorical diagnosis of $\mathrm{CD}$, the Hyperactivity/Inattention scale has been constructed to mirror hyperactivity, impulsivity, and inattention problems, i.e., core symptoms of ADHD.

We used the Conduct problems scale (five questions), referred to as $C D$ scale in this study, to assess CD-like symptoms, and the Hyperactivity/Inattention scale (five questions), referred to as $A D H D$ scale in this study, to assess ADHD symptoms. In follow-up analyses, the $A D H D$ scale was divided into Hyperactivity/Impulsivity subscale (three questions) and Inattention subscale (two questions), as it has been in previous studies [52]. The CD scale-and not the Emotion problems scale-was used because we focused on the relation between ADHD and CD on a trait level mirroring previous comparisons between the two phenotypes on a categorical diagnostic level [6]. Also, we argue that the $C D$ scale better represents problems of emotional instability, rather than a more sustained low level of mood as in the Emotion problems scale-which is better used to assess highly heterogeneous problems of depression and anxiety.

Each question of the SDQ yields scores from zero to two points. We combined self-rate and parent score (for the relation between the scores see Supplementary Fig. 2), since externalizing problems is assumed to be better assessed by parents, while internalizing problems may be better assessed by the adolescent him/herself.

\section{Magnetic resonance imaging}

Full details of the magnetic resonance imaging acquisition protocols and quality control have been described elsewhere $[47,53]$. In addition to gray matter volume (GMV), we measured cortical thickness (CT) and surface area (SA) as they independently build up the GMV measure and follow separate and independent developmental trajectories $[54,55]$. The structural measures were obtained from $\mathrm{T} 1$ weighted images using the semi-automated segmentation and cortical surface reconstruction methods provided by FreeSurfer v5. [47, 53, 56-61]. In brief, the procedure 
includes intensity normalization, removal of non-brain tissue, segmentation of cortical gray, subcortical white, deep gray matter volumetric structures, as well as triangular tessellation of the gray/white matter interface and white matter/cerebrospinal fluid boundary (pial surface). FreeSurfer also allows the automated parcellation of the cortical surfaces into anatomical regions of interest (ROI), 34 per hemisphere [62]. For each ROI cortical measures were obtained from subjects' native space. Based on our hypothesis, we focussed on four different regions in the main analysis: lateral orbitofrontal cortex (lOFC), rostral and caudal anterior cingulate cortex (rACC and cACC), as well as dorsolateral/dorsomedial prefrontal cortex (dl/ dmPFC). The dl/dmPFC was constructed by combining average cortical volumes extracted from superior frontal, caudal middle frontal and rostral middle frontal cortex, as done in previous studies [63]. All the ROIs were bilateral as we had no unilateral hypothesis.

The statistical approach and discussion on dmPFC and vlPFC in relation to choice of ROIs is described in Supplementary Fig. 3. In brief, we controlled for sex and imaging center in all structural analyses. We used ADHD and $\mathrm{CD}$ scores separately to determine all variance in structure that could account for each trait alone. We also used both scores as independent variables in the same statistical model to determine variance in structure that could be uniquely explained by each of the ADHD and CD scores alone.

We did not correct for head size, since we were interested in a dysfunction of a variety of behaviors that depend on large scale prefrontal networks (as opposed to one specific function). In addition, wide spread cortical alterations associated with ADHD in children have been shown [30]. A correction of head size would therefore partly correct for the measure of interest. Furthermore, in studies focusing on neurodevelopmental disorders, such as ADHD, a correction for IQ is not recommended, since it may also correct for characteristics of the disorder [64]. This reasoning is also relevant for subclinical ADHD traits. We have therefore not corrected for IQ in the main analyses. However, for completeness we provide the results obtained when correcting for intra-cranial volume (ICV) and IQ in the Supplementary Information.

A vertex-wise approach was used to explore more focal associations between the behavioral data and cortical volume that might not be detectable with the ROI approach within the predefined ROIs. In addition, we performed an explorative vertex-wise analysis over the whole brain.

\section{Behavioral tasks}

We analyzed tests that were associated with either "cool" EF, i.e., the Stop Signal Test $[65,66]$ and Spatial Working
Memory (SWM) task [67-69], or with "hot" EF, i.e., Delay Discounting (DD) [70]. The statistical approach is described in Supplementary Fig. 3.

\section{Results}

\section{Descriptive data of ADHD and CD scores}

Residual error revealed normal distributions in all analyses performed. Both the ADHD (mean 6.90 \pm 3.75 ) and CD (mean $3.70 \pm 2.60$ ) trait scores displayed a wide, but leftskewed distribution (Fig. 1a, b). A correlational analysis disclosed a moderate correlation $(r=0.55, p<0.001)$ between the ADHD and the CD scores (Fig. 1c).

\section{MR data (ROI-analysis)}

\section{Individual effects of $A D H D$ and $C D$ on brain structure}

Both ADHD scores and CD scores correlated negatively with gray matter volumes (GMVs) and surface area (SA) in all ROIs (cACC, dl/dmPFC, 1OFC, and rACC), while cortical thickness (CT) did not show any correlation with these traits (Table 1; Supplementary Fig. 4).

\section{Specific effects of ADHD and CD on brain structure}

GMV in dl/dmPFC and cACC correlated negatively with the ADHD scores after controlling for CD scores, however this effect did not reach significance after correction for the number of tests (Table $2 \mathrm{a}$ ). SA in $\mathrm{dl} / \mathrm{dmPFC}$ and $\mathrm{cACC}$ correlated negatively with the ADHD scores after controlling for CD scores (Table 2b). There was no significant relation between ADHD scores and CT in any of the ROIs (Table 2c).

There was a significant negative correlation between GMV in rACC and CD scores when controlling for ADHD scores (Table 3a). No significant effect of CD scores was observed in 1OFC GMV when controlling for ADHD scores. There was a negative correlation between the SA and $\mathrm{CD}$ scores in $\mathrm{AACC}$ after controlling for ADHD scores on a threshold-significant level (Table 3b). No significant effect was observed between the $\mathrm{CD}$ scores and SA in 1OFC after controlling for ADHD scores. There was no significant relation between $\mathrm{CT}$ of the predefined ROIs and CD scores (Table 3c). A 3D-representation of the relationships between the two behavioral dimensions (ADHD and CD trait) and GMV in the ACC ROIs is shown in Fig. 2.

We had no hypotheses that there would be any specific negative correlations between IOFC / rACC and ADHD scores, or between $\mathrm{cACC} / \mathrm{dl} / \mathrm{dmPFC}$ and $\mathrm{CD}$ scores. 

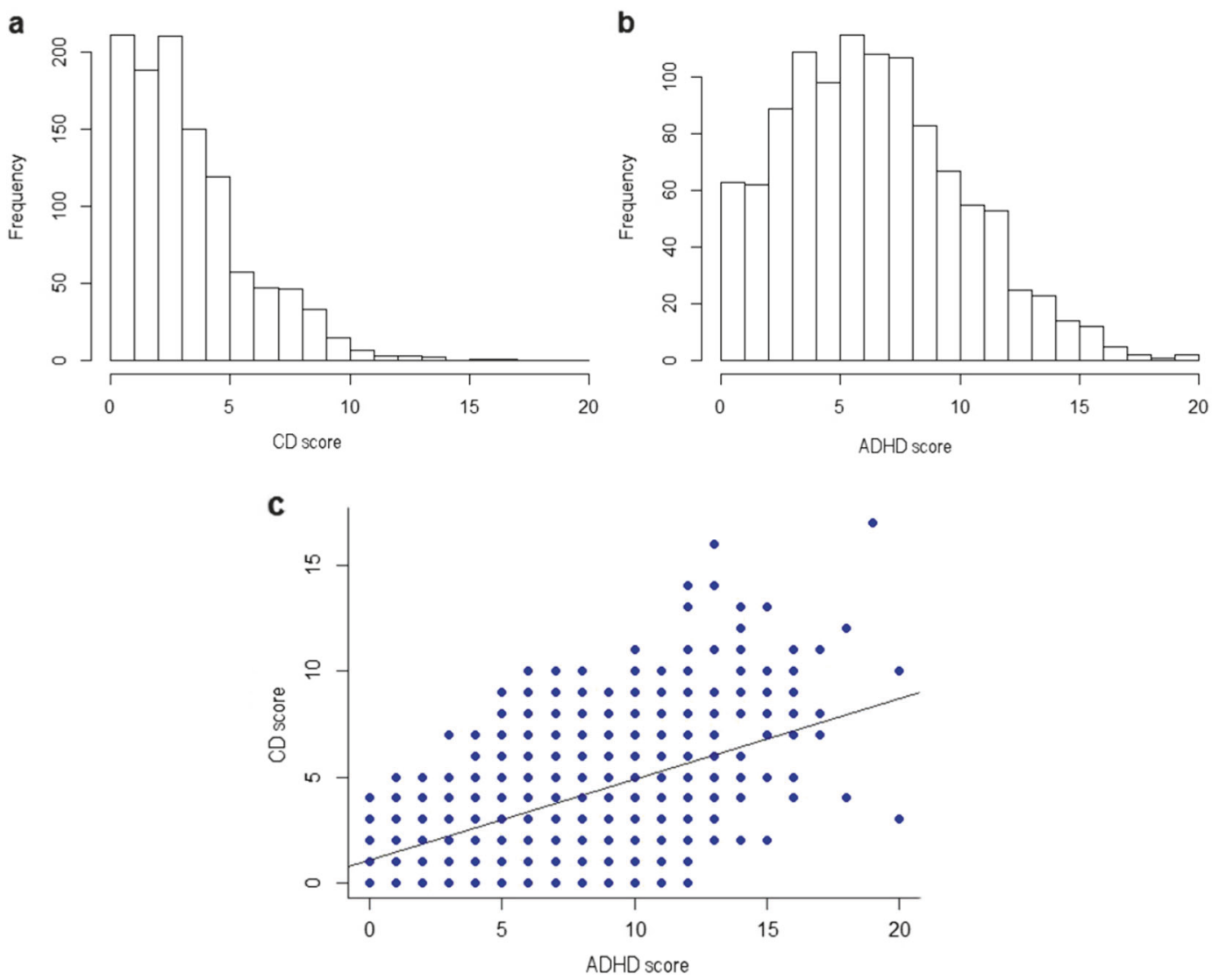

Fig. 1 Description of SDQ distribution in our sample of the CD (Conduct) and ADHD (Hyperactivity/Inattention) scores (a and b) and the correlation $(r=0.549, p<0.001)$ between the two main subscales $(\mathbf{c})$

However, the results of those analyses are shown in Supplementary Tables 2 and 3 for completeness.

\section{Exploratory analysis}

A vertex-wise analysis within the ROIs under investigation (Fig. 3) also suggests more focal/localized effects. Moreover, it confirmed the extensive overlap between the effect of ADHD and the effect of CD on brain structure suggested in the ROI-analysis (Fig. 3).

In the whole brain analysis (vertex-wise approach, cluster-wise corrected) we observed similar significant and largely overlapping negative relations between (a) the ADHD trait and GMV, and (b) CD trait and GMV in ACC and prefrontal cortex (Fig. 4). We also observed that the ADHD trait was significantly related to smaller GMV in several regions such as temporopolar and orbitofrontal cortex after controlling for CD trait, while the CD trait was more related to smaller GMV in left temporoparietal junction after controlling for ADHD trait (Fig. 4).

In order to understand whether hyperactivity or inattention problems better explained the relation between ADHD traits and brain structure we divided the $A D H D$ scale into
Hyperactivity/Impulsivity scores and Inattention scores. This analysis showed that both Hyperactivity/Impulsivity scores and Inattention scores contributed to the structural effects in cACC and dl/dmPFC (Supplementary Table 4). However, this contribution was larger for the Hyperactivity/ Impulsivity scores.

Similar structural findings as in the main analysis, albeit to a lower significance level, were observed for ADHD scores and for CD scores when controlling for ICV and IQ (Supplementary Tables 5 and 6).

When studying only a sub-population of the subjects with a low indication of a psychiatric diagnosis $(n=860)$ similar general result were observed for the ADHD trait (Supplementary Table 7). The effects of the CD trait were weaker in this analysis with a border significant negative correlation in the main analysis between CD trait and GMV in rACC.

Our exploratory results also suggest that although both higher ADHD and CD scores are related to a smaller GMV in dmPFC, this effect still remains for the ADHD trait when controlling for the CD trait (Supplementary Table 8; Fig. 3). Moreover, neither the ADHD trait nor the $\mathrm{CD}$ trait was significantly related to vlPFC morphology (Supplementary Table 8). 
Table 1 Main effects of ADHD and $\mathrm{CD}$ scores on gray matter volume (GMV; a), surface area (SA; b) and cortical thickness $(\mathrm{CT} ; \mathrm{c})$ of the pre-defined ROIs when not controlling for the other trait dimension
Table 2 Testing specific hypothesis 1

\begin{tabular}{lll}
\hline & ADHD score & CD score \\
\hline ROI GMV (a) & & \\
cACC & $-0.104, p<0.001^{\mathrm{a}}, R^{2}=0.088$ & $-0.099, p<0.001^{\mathrm{a}}, R^{2}=0.087$ \\
dl/dmPFC & $-0.086, p<0.001^{\mathrm{a}}, R^{2}=0.326$ & $-0.075, p=0.003^{\mathrm{a}}, R^{2}=0.325$ \\
IOFC & $-0.108, p>0.001^{\mathrm{a}}, R^{2}=0.295$ & $-0.073, p=0.005^{\mathrm{a}}, R^{2}=0.289$ \\
rACC & $-0.095, p=0.001^{\mathrm{a}}, R^{2}=0.117$ & $-0.104, p<0.001^{\mathrm{a}}, R^{2}=0.119$ \\
ROI SA (b) & & \\
cACC & $-0.116, p<0.001^{\mathrm{a}}, R^{2}=0.113$ & $-0.100, p<0.001^{\mathrm{a}}, R^{2}=0.110$ \\
dl/dmPFC & $-0.097, p<0.001^{\mathrm{a}}, R^{2}=0.338$ & $-0.078, p=0.002^{\mathrm{a}}, R^{2}=0.335$ \\
IOFC & $-0.111, p<0.001^{\mathrm{a}}, R^{2}=0.227$ & $-0.067, p=0.013^{\mathrm{a}}, R^{2}=0.220$ \\
rACC & $-0.102, p<0.001^{\mathrm{a}}, R^{2}=0.147$ & $-0.099, p<0.001^{\mathrm{a}}, R^{2}=0.147$ \\
ROI CT (c) & & \\
cACC & $0.009, p=0.748, R^{2}=0.158$ & $0.018, p=0.528, R^{2}=0.158$ \\
dl/dmPFC & $-0.017, p=0.554, R^{2}=0.088$ & $-0.013, p=0.668, R^{2}=0.088$ \\
lOFC & $-0.028, p=0.291, R^{2}=0.245$ & $-0.019, p=0.485, R^{2}=0.244$ \\
rACC & $0.016, p=0.588, R^{2}=0.129$ & $0.005, p=0.857, R^{2}=0.129$ \\
\hline
\end{tabular}

Values are standardized $\beta$-weights. $N=1093$

GMV: Average intra-correlation coefficient $=0.516$, adjusted $p$-level $=0.026$. SA: Average intra-correlation coefficient $=0.633$, adjusted $p$-level $=0.030$. CT: Average intra-correlation coefficient $=0.275$, adjusted $p$-level $=0.019$

andicates significance after correction for multiple comparisons, based on the average intra-correlation coefficient between all four ROIs

\begin{tabular}{cll}
\hline & ADHD score & ADHD score controlling for CD score \\
\hline ROI GMV (a) & & \\
cACC & $-0.104, p<0.001^{\mathrm{a}}, R^{2}=0.088$ & $-0.071, p=0.047^{\mathrm{b}}, R^{2}=0.090$ \\
dl/dmPFC & $-0.086, p<0.001^{\mathrm{a}}, R^{2}=0.326$ & $-0.064, p=0.037^{\mathrm{b}}, R^{2}=0.327$ \\
ROI SA (b) & & \\
cACC & $-0.116, p<0.001^{\mathrm{a}}, R^{2}=0.113$ & $-0.087, p=0.013^{\mathrm{a}}, R^{2}=0.115$ \\
dl/dmPFC & $-0.097, p<0.001^{\mathrm{a}}, R^{2}=0.338$ & $-0.077, p=0.011^{\mathrm{a}}, R^{2}=0.339$ \\
ROI CT (c) & & \\
cACC & $0.009, p=0.748, R^{2}=0.158$ & $-0.001, p=0.972, R^{2}=0.158$ \\
dl/dmPFC & $-0.017, p=0.554, R^{2}=0.088$ & $-0.015, p=0.671, R^{2}=0.088$ \\
\hline
\end{tabular}

(a) ROI gray matter volume (GMV) regressed on ADHD scores with and without controlling for CD scores. (b) ROI surface area (SA) regressed on ADHD scores with and without controlling for CD scores. (c) ROI cortical thickness (CT) regressed on ADHD scores with and without controlling for CD scores. There were no significant effects for the cortical thickness (CT) analysis. Values are given in standardized $\beta$-weights

GMV: Average intra-correlation coefficient $=0.424$, adjusted $p$-level $=0.034$. SA: Average intra-correlation coefficient $=0.594$, adjusted $p$-level $=0.038$. CT: Average intra-correlation coefficient $=0.115$, adjusted $p$ level $=0.027$

andicates significance after correction for multiple comparisons, based on the average intra-correlation coefficient between cACC and $\mathrm{dl} / \mathrm{dmPFC}$

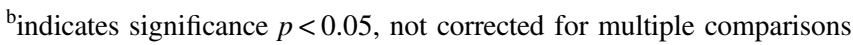

\section{Behavioral results}

\section{Stop Signal Test-SDQ}

ADHD score correlated with SSRT (standardized $\beta=$ 0.099, $p=0.021$ ), while CD score did not (standardized $\beta=0.014, p=0.740)$. When controlling for CD score, the ADHD score uniquely explained a significant amount of the SSRT variance (standardized $\beta=0.131, \quad p=0.011$ ). Exploratory analysis suggested that this correlation was driven by the Inattention subscore, which uniquely explained a significant part of the variance when controlling 
Table 3 Testing specific hypothesis 2
CD score

CD score controlling for ADHD score

\begin{tabular}{ll}
\hline ROI GMV (a) & \\
IOFC & $-0.073, p=0.005^{\mathrm{a}}, R^{2}=0.289$ \\
rACC & $-0.104, p<0.001^{\mathrm{a}}, R^{2}=0.119$ \\
ROI SA (b) & \\
IOFC & $-0.067, p=0.013^{\mathrm{a}}, R^{2}=0.220$ \\
rACC & $-0.099, p<0.001^{\mathrm{a}}, R^{2}=0.147$ \\
ROI CT (c) & \\
IOFC & $-0.019, p=0.485, R^{2}=0.244$ \\
rACC & $0.005, p=0.857, R^{2}=0.129$
\end{tabular}

$$
\begin{aligned}
& -0.020, p=0.523, R^{2}=0.295 \\
& -0.076, p=0.029^{\mathrm{a}}, R^{2}=0.121 \\
& -0.009, p=0.782, R^{2}=0.227 \\
& -0.062, p=0.067, R^{2}=0.150 \\
& -0.004, p=0.893, R^{2}=0.245 \\
& -0.005, p=0.884, R^{2}=0.129
\end{aligned}
$$

(a) ROI gray matter volume (GMV) regressed on CD scores with and without controlling for ADHD scores. b) ROI surface area (SA) regressed on CD scores with and without controlling for ADHD scores. (c) ROI cortical thickness (CT) regressed on CD scores with and without controlling for ADHD scores. Values given are standardized $\beta$-weights

aindicates significance after correction for multiple comparisons, based on the average intra-correlation coefficient between 1OFC and rACC. GMV: Average intra-correlation coefficient $=0.518$, adjusted $p$-level $=0.036$. SA: Average intra-correlation coefficient $=0.593$, adjusted $p$-level $=0.038$. CT: Average intra-correlation coefficient $=0.249$, adjusted $p$-level $=0.030$
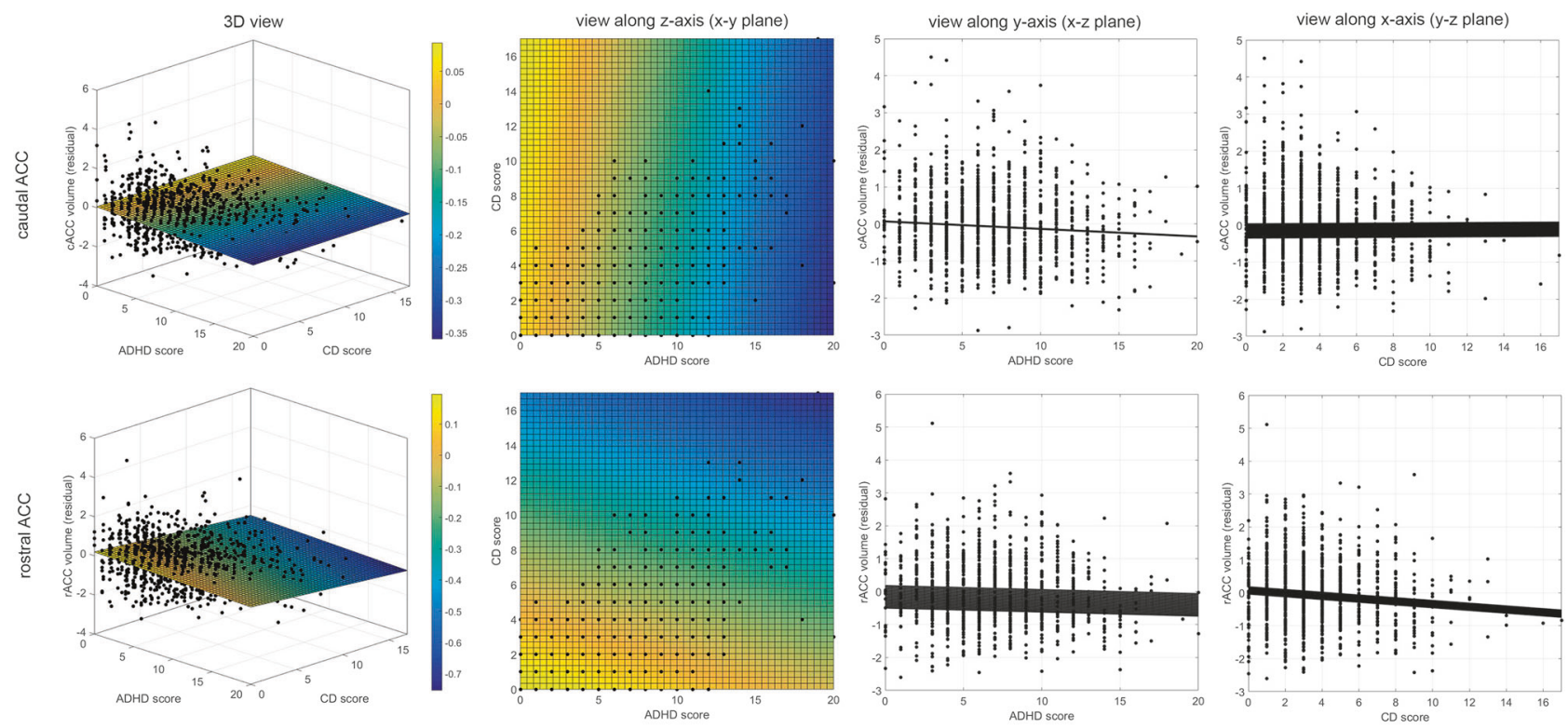

Fig. 2 3D-representation of the relationships between the two behavioral dimensions-ADHD scores ( $x$-axis) and CD scores ( $y$-axis) - and the residuals of the dependent variable GMV (z-axis), after adjusting for sex and center as obtained from the main analysis. Two representative examples for the rACC volume (bottom) and cACC volume (top) are shown. Respective views along the $z$-axis (onto the $x-y$ plane), $y$-axis (onto $x-z$ plane) and $x$-axis (onto $x-z$ plane) are shown, displaying the mutual relationships between two measures. For illustrative purpose, the 3D-data was fitted with a two-dimensional linear robust regression using a polynomial according to the equation. Volume $=a+b \times A D H D$ score $+c \times$ $C D$ score. To better visualize the angle of the obtained plane, its height along the $z$-axis (volume) has been color-coded with warm colors (yellow) indicating larger volumes, and cold colors (blue) representing lower volumes. In case of cACC, the color gradient changes towards the ADHD score dimension, whereas in case of rACC the gradient direction is more shifted towards the CD score dimension. This reflects the discussed associations between $\mathrm{CACC}$ and ADHD score after correcting for CD score, and between rACC and CD scores after correcting for ADHD scores as presented in the main analysis

for Hyperactivity/Impulsivity subscore and $\mathrm{CD}$ score (standardized $\beta=0.158, p=0.003$ ). Neither the ADHD nor CD scores correlated with GoRT (ADHD score: standardized $\beta=0.040, \quad p=0.343 ; \quad \mathrm{CD}$ score: standardized $\beta=0.028, p=0.494)$ ). Results are shown in Table 4a.

\section{Spatial Working Memory task-SDQ}

Both ADHD and CD scores correlated with number of errors in the SWM task (ADHD score: standardized $\beta=0.167, p<0.001 ; \mathrm{CD}$ score: standardized $\beta=0.149$, 


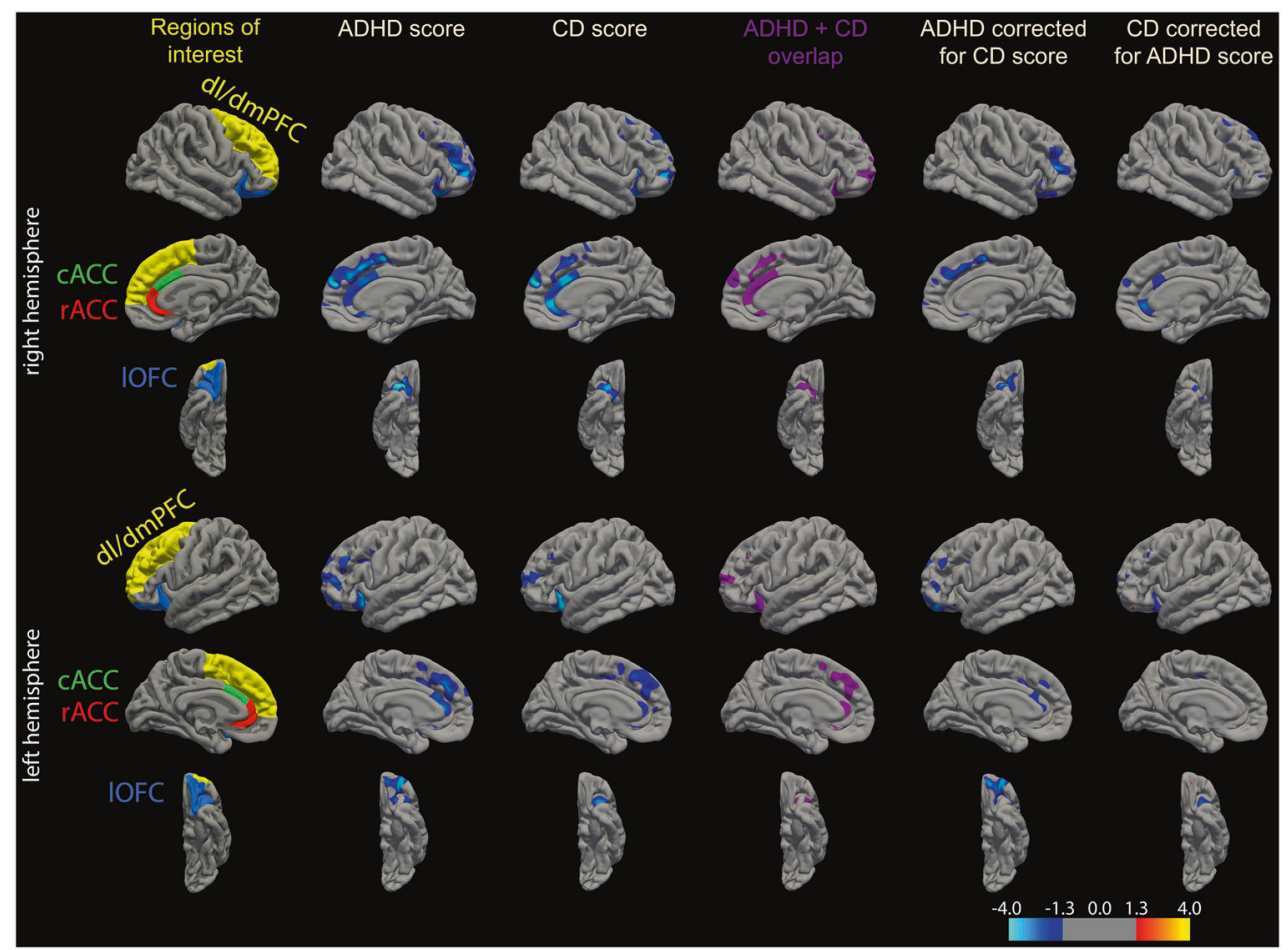

Fig. 3 ROIs as defined by the Desikan atlas that are used for the main analysis are shown in the left column. The other columns contain results of the exploratory analysis showing correlations within those ROIs on a vertex-level. In column 2-3 correlations between GMV and ADHD and CD scores, respectively, are shown. Their regional overlap is shown in column 4 (purple areas). Both ADHD and CD score revealed negative correlations with GMV within a sub-region of the left and right $\mathrm{dl} / \mathrm{dmPFC}$. Those were mainly localized in a cluster comprising the rostral middle frontal and lateral superior frontal cortex, extending to medial portions of the prefrontal cortex. Similar focal associations were observed in posterior and anterior sub-regions of the left and right 1OFC. Correlations observed in the ACC were spread over right caudal and rostral ACC ROIs, whereas a correlation of more focal nature was observed in the left ACC. When correcting for CD trait, the ADHD scores were negatively correlated with the GMV in right rostral middle frontal cortex, right rostral dmPFC, left cACC and bilateral anterior 1OFC (column 5). In contrast, when correcting for ADHD trait, CD scores were negatively correlated with the GMV in right rACC (column 6). Moreover, right cACC and posterior lOFC also showed this relation to a certain extent (column 6). Thus, on a vertex-level there seemed to be a more complex and unique relation between local GMV and the different traits: While GMV in left cACC was more negatively related to ADHD scores, GMV in right cACC and rACC was more related to CD scores. Likewise, while the negative relation between ADHD scores in lOFC GMV was more widespread and anterior when controlling for $\mathrm{CD}$, the negative relation between CD scores in 1OFC GMV was more ventrolateral (spreading into ventrolateral PFC). Overall, no positive correlations were observed. Significance is represented on a $\log (p$-value) scale, where positive values (warm colors) are assigned to positive associations, and negative values (cold colors) to negative associations. For explorative reasons the display threshold was set to 1.3 , corresponding to $p=.05$ (uncorrected)

$p<0.001)$. The ADHD score correlated with the number of errors in the SWM task, when controlling for CD (standardized $\beta=0.120, p<0.001$ ). This correlation seemed to be driven by the Inattention SDQ subscore (standardized $\beta=0.152, p<.001$ ) when controlling for Hyperactivity/ Impulsivity and CD score. The CD score still correlated with the number of errors when controlling for ADHD score (standardized $\beta=0.083, p=0.021$ ). Results are shown in Table 4b.

\section{Delay discounting-SDQ}

Both ADHD and CD scores correlated with the logtransformed k-coefficient from the Delay discounting (DD) task (ADHD score: standardized $\beta=0.139, p<0.001$; CD score: standardized $\beta=0.140, p<0.001)$. The $\mathrm{CD}$ score correlated with $k$ when controlling for ADHD score (standardized $\beta=0.093, p=0.014$ ). ADHD score also explained a unique part of the variance when controlling for CD score (standardized $\beta=0.088, p=0.021$ ). Results are shown in Table 4c.

\section{Exploratory analysis}

In an exploratory analysis, we investigated whether information from brain structure could explain variability on ADHD and CD scores beyond that explained by behavioral tests alone. The analysis showed that SSRT and brain structure related to non-emotional top-down control (cACC and $\mathrm{dl} / \mathrm{dmPFC}$ GMV, as well as $\mathrm{SA}$ ) each uniquely explained a part of the variance of the ADHD score (Supplementary Table 9), while Delay discounting kcoefficient and brain structure related to emotional topdown control (rACC GMV, as well as SA) each uniquely 


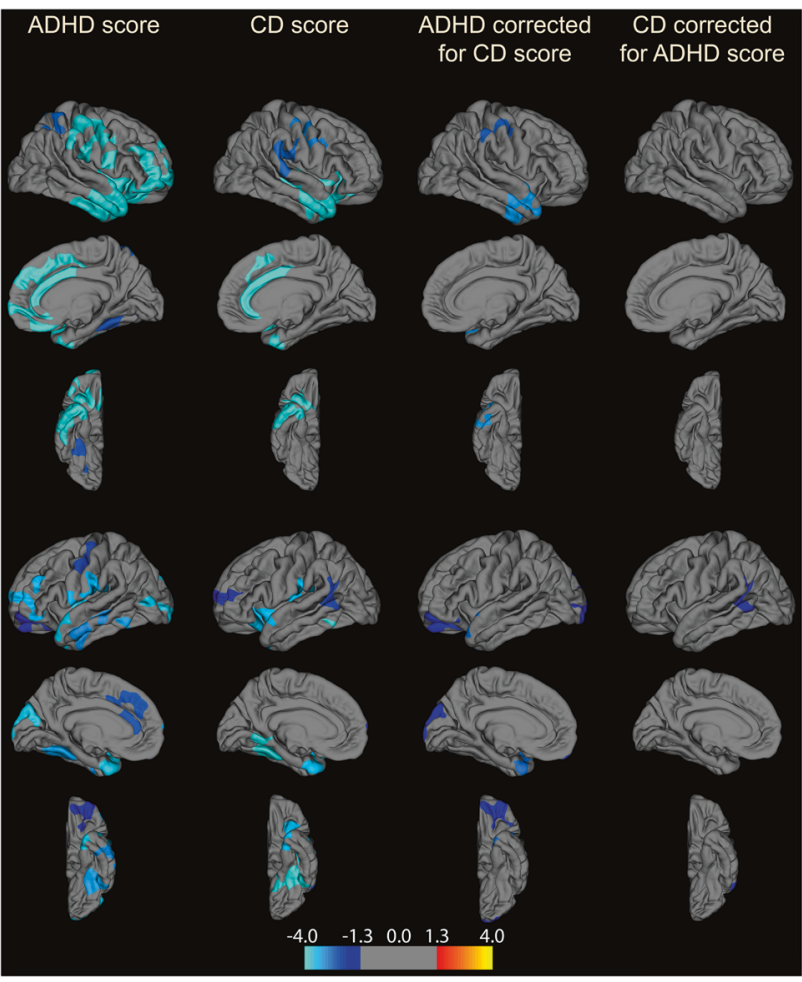

Fig. 4 An exploratory vertex-wise analysis was performed calculating correlations between GMV and ADHD/CD scores across the whole brain. Multiple comparison correction was done using a Monte Carlo cluster wise approach (cluster threshold $p<0.05$ ). Both ADHD and $\mathrm{CD}$ scores were significantly related to smaller GMV in frontal brain region clusters (d1/dmPFC, rostral/caudal ACC, and lOFC). Additional clusters revealing significant negative correlations outside our ROI:s were also observed: ADHD scores were negatively correlated to GMV in right anterior insula, bilateral inferior frontal cortex (pars orbitalis), bilateral temporal cortex, bilateral precentral, and postcentral (motor/ sensory) cortex, right medial OFC, left medial and lateral occipital cortex, and bilateral fusiform. When correcting for CD scores clusters in bilateral temporal/temporopolar regions, left medial and lateral occipital cortex, right precentral and postcentral, as well as left lOFC were still present. CD scores showed additional negative correlations in precentral and postcentral cortex, anterior temporal cortex, bilateral anterior insula, left fusiform cortex, and bilateral temporo-parietal junction (TPJ), which, except for the cluster in left TPJ, disappeared when correcting for ADHD scores. Significance is represented on a log ( $p$-value) scale, where positive values (warm colors) are assigned to positive associations, and negative values (cold colors) to negative associations. Right hemisphere is shown on the top and left hemisphere is shown on the bottom in the figure

explained part of the CD score variance (Supplementary Table 10).

\section{Discussion}

In the present study, we showed that both ADHD and CD trait scores correlated negatively with gray matter volume (GMV) and surface area (SA) in cACC, $\mathrm{dl} / \mathrm{dmPFC}, \mathrm{rACC}$, and IOFC in a large community sample of adolescents using a ROI approach. As hypothesized, there was a substantial overlap between the effects of the ADHD and the CD traits on brain morphology (Fig. 3). Thus, investigating ADHD symptoms without considering $\mathrm{CD}$ symptoms, or vice versa, makes it difficult to distinguish between their unique effects on brain structure. Our analyses indicated that cACC and $\mathrm{d} l / \mathrm{dmPFC}$ SA correlated negatively with ADHD score after controlling for CD score. Similar findings were observed for GMV, although these did not survive correction for multiple comparisons. In contrast, $\mathrm{CD}$ score correlated negatively with the GMV in rACC after controlling for ADHD score (SA results on trend-level). Our study suggests that, apart from the large overlap in structural relations, there are morphological characteristics that are specific to either ADHD symptoms or CD symptoms, and that those relations can be observed on trait level within a community sample.

The current findings are in line with the idea that nonemotional ADHD symptoms are associated with a smaller structure [30-33] and lower function [26-29] of cACC and $\mathrm{dl} / \mathrm{dmPFC}$, areas that have been linked to "cool" EF $[24,25]$, while emotional instability and CD symptoms are associated with smaller structure [42-44, 45, 46] and lower function [36, 37, 40, 41, 71, 72] of rACC and bordering areas, that are linked to "hot" EF [73-77] and emotional regulation [49, 78, 79]. While these findings corroborate several previous studies on ADHD and CD patients they also suggest that some structural differences are unique to the two different traits. However, we could not find any evidence for the hypothesized relationship between IOFC morphology and CD trait scores, instead we observed more localized associations in the exploratory vertex-level analysis (Fig. 3). One plausible explanation for this is that the lOFC is still under substantial development during adolescence [80] and that this relationship might be detectable only later in life.

Previous studies of inattention/hyperactivity and emotional instability have typically focused on categorically defined patient groups in the respective domains, such as ADHD or BPD, ASPD, and CD. However, it has been shown that subclinical symptoms associated with inattention or emotional instability also occur in the general population to a variable degree, often following a normal or seminormal distribution [2, 14-16]. It has been hypothesized that patients represent extreme cases within this symptom distribution [2]. This variability may occur due to different capacities in the underlying regulatory systems in the brain, i.e., cognitive core capacities [2]. On a whole this model posits that disorders involving top-down dysregulation, such as ADHD or BPD, ASPD, and CD, are better understood using a dimensional model rather than a categorical model [2]. Our findings that dimensional measures of ADHDsymptoms and $\mathrm{CD}$-symptoms in a general population are 
Table 4 Behavioral tests and results

\begin{tabular}{lllll}
\hline Correlation with SDQ & ADHD score & ADHD score controlling for CD & CD score & CD score controlling for ADHD \\
\hline Stop Signal test (SSRT) (a) & $\beta=0.099$ & $\beta=0.131$ & $\beta=0.014$ & na \\
& $p=0.021$ & $p=0.011$ & $p=0.740$ & $\beta=0.083$ \\
Spatial Working Memory & $\beta=0.167$ & $\beta=0.120$ & $\beta=0.149$ & $p=0.021$ \\
task (number of errors) (b) & $p<0.001$ & $p<0.001$ & $p<0.001$ & $\beta=0.093$ \\
Delay discounting (k- & $\beta=0.139$ & $\beta=0.088$ & $\beta=0.140$ & $p=0.014$ \\
coefficient) (c) & $p<0.001$ & $p=0.021$ & $p<0.001$ & $p$ \\
\hline
\end{tabular}

(a) The Stop Signal Test [66] was used to assess impulsivity control. From this an estimated Stop Signal Reaction Time (SSRT) was obtained $[65,66]$. A longer SSRT indicates higher impulsivity, and a difficulty to stop a response. An average baseline reaction time, GoRT, was also calculated for each subject. Due to technical difficulties sufficient data was obtained from 563 of the selected subjects. A significant correlation between SSRT and ADHD scores was observed. This remained significant when controlling for CD scores. No correlation with CD scores was found. (b) The Spatial Working Memory (SWM) task was used to assess working memory [67-69]. In this task, a number of errors is obtained for each participant, where a higher number of errors corresponds to poorer working memory performance. Sufficient data was obtained from 1056 of the selected subjects. Both ADHD and CD scores correlated with the number of errors, even after controlling for the other dimension (c). Delay discounting (DD) task was used to assess emotional impulsiveness [70] related to emotional dysregulation [2]. In DD an estimated "discount coefficient" $\mathrm{k}$ is obtained. A larger k-coefficient reflects a larger delay discounting, meaning valuing a small immediate reward higher than a larger delayed reward. The estimated k-coefficients were log transformed to give more normally distributed data. Data was obtained from 1088 subjects. The statistical approach is described in Supplementary Fig. 3. Both ADHD and CD scores correlated with the "discount coefficient" $k$, even after controlling for the other dimension.

correlated to cognitive function and some of the brain morphology previously shown to be altered in the respective disorders partially support this hypothesis. However, it is important to stress that clinical disorders such as ADHD and CD typically show significantly larger impairment and often include co-morbidities. This may be a reason why some structural findings observed in large meta-analyses were not present on a trait level. Also, as the present study focused on cortical measures it is not known how the traits relate to subcortical structures. It is noteworthy that dmPFC showed a relation to the ADHD trait in the present study although it has not been found to be altered in two recent meta-analysis on ADHD [29, 34]. However, in another meta-analysis a region in $\mathrm{cACC}$ adjacent to and partly overlapping with dmPFC was smaller in adult ADHD patients as compared to controls [33]. It should also be pointed out that dmPFC is related to the term "ADHD" in Neurosynth [81] based on fMRI data. Apart from the present study, two recent studies $[48,82]$ have used a dimensional approach and observed a negative relation between sub-clinical ADHD symptoms and GMV in medial prefrontal annd anterior cinngulate regions. However, these studies have not disentangled non-emotional ADHD traits from emotional instability or CD traits.

The structural findings that are specific to ADHD or CD scores were assumed to be mirrored in the behavioral measurements. We hypothesized that "cool" EF should be associated specifically to ADHD traits, while "hot" EF should be more related to $\mathrm{CD}$ traits. In line with this suggestion, we showed a negative correlation between ADHD score and motor impulse control in the Stop Signal Test, which remained significant when controlling for CD scores. Impaired working memory capacity has also frequently been linked to ADHD problems [3]. Surprisingly, we observed that both the level of ADHD and CD problems uniquely explained part of the working memory performance, making this task less specific to either problem domain. Finally, we observed that both ADHD and CD scores were uniquely related to delay discounting tendency. This was expected, since performance on this task depends on both non-emotional general attentional control, as well as emotionally related regulation of reward processes. This result is in line with previous findings reporting that some ADHD subgroups show suboptimal results in "cool" EF tasks, such as Stop Signal Test and Stroop test, whereas other subgroups show deficits in "hot" EF tasks, such as delay discounting [17]. Interestingly, our data suggests that brain structure explains unique aspects of ADHD and CD traits, beyond what is explained by behavioral results. Thus, a combination of both measurements may be the most optimal marker for these traits.

There are several limitations in the study. First, apart from emotional instability, the CD trait also captures a less common phenotype that includes callous-unemotional and psychopathic behavior associated with different processing of emotional stimuli [4]. Second, although the structural findings related to the ADHD trait were mostly driven by hyperactivity/impulsiveness questions, the behavioral findings related to the ADHD trait were mostly driven by the inattention questions. Thus, future studies should further investigate the inter-relationships between the specific ADHD sub-traits, structural and cognitive characteristics. Third, ADHD has been associated with different types of emotional dysregulation symptoms [19]. Future studies should investigate how such dysregulations are related to more specific features of CD traits. Finally, as common in studies on hyperactivity, movement artefacts may have 
partly contributed to the structural findings. However, these seem to be mediated by changes in cortical thickness [83] where we did not observe any associations with ADHD traits.

Acknowledgements This work received support from the following sources: the European Union-funded FP6 Integrated Project IMAGEN (Reinforcement-related behavior in normal brain function and psychopathology) (LSHM-CT- 2007-037286), the Horizon 2020 funded ERC Advanced Grant 'STRATIFY' (Brain network based stratification of reinforcement-related disorders) (695313), ERANID (Understanding the Interplay between Cultural, Biological and Subjective Factors in Drug Use Pathways) (PR-ST-0416-10004), BRIDGET (JPND: BRain Imaging, cognition Dementia and next generation GEnomics) (MR/N027558/1), the FP7 projects IMAGEMEND (602450; IMAging GEnetics for MENtal Disorders) and MATRICS (603016), the Innovative Medicine Initiative Project EU-AIMS (115300-2), the Medical Research Council Grant 'c-VEDA' (Consortium on Vulnerability to Externalizing Disorders and Addictions) (MR/N000390/1), the Swedish Research Council FORMAS, the Medical Research Council, the National Institute for Health Research (NIHR) Biomedical Research Centre at South London and Maudsley NHS Foundation Trust and King's College London, the Bundesministeriumfür Bildung und Forschung (BMBF grants 01GS08152; 01EV0711; eMED SysAlc01ZX1311A; Forschungsnetz AERIAL), the Deutsche Forschungsgemeinschaft (DFG grants SM 80/7-1, SM 80/7-2, SFB 940/1). Further support was provided by grants from: ANR (project AF12-NEUR0008-01-WM2NA, and ANR-12-SAMA0004), the Fondation de France, the Fondation pour la Recherche Médicale, the Mission Interministérielle de Lutte-contre-les-Drogueset-les-Conduites-Addictives (MILDECA), the Assistance-PubliqueHôpitaux-de-Paris and INSERM (interface grant), Paris Sud University IDEX 2012; the National Institutes of Health, Science Foundation Ireland (16/ERCD/3797), U.S.A. (Axon, Testosterone and Mental Health during Adolescence; RO1 MH085772-01A1), and by NIH Consortium grant U54 EB020403, supported by a cross-NIH alliance that funds Big Data to Knowledge Centres of Excellence. Also grants from Karolinska Institutet, (Karolinska Institutet Strategic Neuroscience Program, Clinical Scientist Training Programme (CSTP), Senior Researcher in Medical Science), Stockholm County Council (PPG project), Stockholm Swedish Society of Medicine, Söderström-Königska Foundation and Osher Center for Integrative Medicine supported this study.

\section{Compliance with ethical standards}

Conflict of interest Dr. TB served in an advisory or consultancy role for Actelion, Hexal Pharma, Lilly, Lundbeck, Medice, Novartis, Shire. He received conference support or speaker's fee by Lilly, Medice, Novartis, and Shire. He has been involved in clinical trials conducted by Shire and Viforpharma. He received royalities from Hogrefe, Kohlhammer, CIP Medien, Oxford University Press. The present work is unrelated to the above grants and relationships. Dr. GB has received funding for a $\mathrm{PhD}$ student and honoraria for teaching on scanner programming courses from General Electric Healthcare; he acts as a consultant for IXICO. Dr. HW received a speaker honorarium from Servier (2014). The remaining authors declare no conflict of interest.

\section{References}

1. Barkley RA. Behavioral inhibition, sustained attention, and executive functions: constructing a unifying theory of ADHD. Psychol Bull. 1997;121:65-94.
2. Petrovic P, Castellanos FX. Top-down dysregulation-from ADHD to emotional instability. Front Behav Neurosci. 2016;10:1-25.

3. Willcutt EG, Doyle AE, Nigg JT, Faraone SV, Pennington BF. Validity of the executive function theory of attention-deficit/ hyperactivity disorder: a meta-analytic review. Biol Psychiatry. 2005;57:1336-46.

4. Blair RJ, Leibenluft E, Pine DS. Conduct disorder and callousunemotional traits in youth. N Engl J Med. 2014;371:2207-16.

5. Glenn AL, Johnson AK, Raine A. Antisocial personality disorder: a current review. Curr Psychiatry Rep. 2013;15:427.

6. Rubia K. "Cool" inferior frontostriatal dysfunction in attentiondeficit/hyperactivity disorder versus "hot" ventromedial orbitofrontal-limbic dysfunction in conduct disorder: a review. Biol Psychiatry. 2011;69:e69-87.

7. Sebastian A, Jung P, Krause-Utz A, Lieb K, Schmahl C, Tuscher O. Frontal dysfunctions of impulse control - a systematic review in borderline personality disorder and attention-deficit/hyperactivity disorder. Front Hum Neurosci. 2014;8:698.

8. Anckarsater H, Stahlberg O, Larson T, Hakansson C, Jutblad SB, Niklasson L, et al. The impact of ADHD and autism spectrum disorders on temperament, character, and personality development. Am J Psychiatry. 2006;163:1239-44.

9. Philipsen A, Limberger MF, Lieb K, Feige B, Kleindienst N, Ebner-Priemer U, et al. Attention-deficit hyperactivity disorder as a potentially aggravating factor in borderline personality disorder. Br J Psychiatry. 2008;192:118-23.

10. Semiz UB, Basoglu C, Oner O, Munir KM, Ates A, Algul A, et al. Effects of diagnostic comorbidity and dimensional symptoms of attention-deficit-hyperactivity disorder in men with antisocial personality disorder. Aust N Z J Psychiatry. 2008;42:405-13.

11. Storebo OJ, Simonsen E. The association between ADHD and Antisocial Personality Disorder (ASPD): a review. J Atten Disord. 2013;20:815-24.

12. Distel MA, Carlier A, Middeldorp CM, Derom CA, Lubke GH, Boomsma DI. Borderline personality traits and adult attentiondeficit hyperactivity disorder symptoms: a genetic analysis of comorbidity. Am J Med Genet B Neuropsychiatr Genet. 2011;156B:817-25.

13. Insel T, Cuthbert B, Garvey M, Heinssen R, Pine DS, Quinn K, et al. Research domain criteria (RDoC): toward a new classification framework for research on mental disorders. Am J Psychiatry. 2010;167:748-51.

14. Crosbie J, Arnold P, Paterson A, Swanson J, Dupuis A, Li X, et al. Response inhibition and ADHD traits: correlates and heritability in a community sample. J Abnorm Child Psychol. 2013;41:497-507.

15. Das D, Cherbuin N, Butterworth P, Anstey KJ, Easteal S. A population-based study of attention deficit/hyperactivity disorder symptoms and associated impairment in middle-aged adults. PLoS ONE. 2012;7:e31500.

16. Petrovic P, Ekman CJ, Klahr J, Tigerstrom L, Ryden G, Johansson $\mathrm{AG}$, et al. Significant grey matter changes in a region of the orbitofrontal cortex in healthy participants predicts emotional dysregulation. Soc Cogn Affect Neurosci. 2015;11:1041-9.

17. Castellanos FX, Sonuga-Barke EJ, Milham MP, Tannock R. Characterizing cognition in ADHD: beyond executive dysfunction. Trends Cogn Sci. 2006;10:117-23.

18. Castellanos FX, Tannock R. Neuroscience of attention-deficit/ hyperactivity disorder: the search for endophenotypes. Nat Rev Neurosci. 2002;3:617-28.

19. Shaw P, Stringaris A, Nigg J, Leibenluft E. Emotion dysregulation in attention deficit hyperactivity disorder. Am J Psychiatry. 2014;171:276-93.

20. Alderson RM, Rapport MD, Kofler MJ. Attention-deficit/hyperactivity disorder and behavioral inhibition: a meta-analytic review 
of the stop-signal paradigm. J Abnorm Child Psychol. 2007;35:745-58.

21. Lansbergen MM, Kenemans JL, van Engeland H. Stroop interference and attention-deficit/hyperactivity disorder: a review and metaanalysis. Neuropsychology. 2007;21:251-62.

22. Nichols SL, Waschbusch DA. A review of the validity of laboratory cognitive tasks used to assess symptoms of ADHD. Child Psychiatry Hum Dev. 2004;34:297-315.

23. Bush G. Cingulate, frontal, and parietal cortical dysfunction in attention-deficit/hyperactivity disorder. Biol Psychiatry. 2011;69:1160-7.

24. Cieslik EC, Mueller VI, Eickhoff CR, Langner R, Eickhoff SB. Three key regions for supervisory attentional control: evidence from neuroimaging meta-analyses. Neurosci Biobehav Rev. 2015;48:22-34

25. Nee DE, Wager TD, Jonides J. Interference resolution: insights from a meta-analysis of neuroimaging tasks. Cogn Affect Behav Neurosci. 2007;7:1-17.

26. Cortese S, Kelly C, Chabernaud C, Proal E, Di Martino A, Milham MP, et al. Toward systems neuroscience of ADHD: a metaanalysis of 55 fMRI studies. Am J Psychiatry. 2012;169:1038-55.

27. Dickstein SG, Bannon K, Castellanos FX, Milham MP. The neural correlates of attention deficit hyperactivity disorder: an ALE metaanalysis. J Child Psychol Psychiatry. 2006;47:1051-62.

28. Hart H, Radua J, Nakao T, Mataix-Cols D, Rubia K. Metaanalysis of functional magnetic resonance imaging studies of inhibition and attention in attention-deficit/hyperactivity disorder: exploring task-specific, stimulant medication, and age effects. JAMA Psychiatry. 2013;70:185-98.

29. Norman LJ, Carlisi C, Lukito S, Hart H, Mataix-Cols D, Radua J, et al. Structural and functional brain abnormalities in attentiondeficit/hyperactivity disorder and obsessive-compulsive disorder: a comparative meta-analysis. JAMA Psychiatry. 2016;73: $815-25$.

30. Shaw P, Eckstrand K, Sharp W, Blumenthal J, Lerch JP, Greenstein D, et al. Attention-deficit/hyperactivity disorder is characterized by a delay in cortical maturation. Proc Natl Acad Sci USA. 2007;104:19649-54.

31. Shaw P, Malek M, Watson B, Greenstein D, de Rossi P, Sharp W. Trajectories of cerebral cortical development in childhood and adolescence and adult attention-deficit/hyperactivity disorder. Biol Psychiatry. 2013;74:599-606.

32. Seidman LJ, Biederman J, Liang L, Valera EM, Monuteaux MC, Brown A, et al. Gray matter alterations in adults with attentiondeficit/hyperactivity disorder identified by voxel based morphometry. Biol Psychiatry. 2011;69:857-66.

33. Frodl T, Skokauskas N. Meta-analysis of structural MRI studies in children and adults with attention deficit hyperactivity disorder indicates treatment effects. Acta Psychiatr Scand. 2012;125:114-26.

34. Nakao T, Radua J, Rubia K, Mataix-Cols D. Gray matter volume abnormalities in ADHD: voxel-based meta-analysis exploring the effects of age and stimulant medication. Am J Psychiatry. 2011;168:1154-63.

35. Enzi B, Doering S, Faber C, Hinrichs J, Bahmer J, Northoff G. Reduced deactivation in reward circuitry and midline structures during emotion processing in borderline personality disorder. World J Biol Psychiatry. 2013;14:45-56.

36. Holtmann J, Herbort MC, Wustenberg T, Soch J, Richter S, Walter $\mathrm{H}$, et al. Trait anxiety modulates fronto-limbic processing of emotional interference in borderline personality disorder. Front Hum Neurosci. 2013;7:54.

37. Jacob GA, Zvonik K, Kamphausen S, Sebastian A, Maier S, Philipsen A, et al. Emotional modulation of motor response inhibition in women with borderline personality disorder: an fMRI study. J Psychiatry Neurosci. 2013;38:164-72.
38. Lang S, Kotchoubey B, Frick C, Spitzer C, Grabe HJ, Barnow S. Cognitive reappraisal in trauma-exposed women with borderline personality disorder. Neuroimage. 2012;59:1727-34.

39. Schulze L, Domes G, Kruger A, Berger C, Fleischer M, Prehn K, et al. Neuronal correlates of cognitive reappraisal in borderline patients with affective instability. Biol Psychiatry. 2011;69:564-73.

40. Silbersweig D, Clarkin JF, Goldstein M, Kernberg OF, Tuescher $\mathrm{O}$, Levy KN, et al. Failure of frontolimbic inhibitory function in the context of negative emotion in borderline personality disorder. Am J Psychiatry. 2007;164:1832-41.

41. Wingenfeld K, Rullkoetter N, Mensebach C, Beblo T, Mertens M, Kreisel S, et al. Neural correlates of the individual emotional Stroop in borderline personality disorder. Psychoneuroendocrinology. 2009;34:571-86.

42. Soloff P, Nutche J, Goradia D, Diwadkar V. Structural brain abnormalities in borderline personality disorder: a voxel-based morphometry study. Psychiatry Res. 2008;164:223-36.

43. Soloff PH, Pruitt P, Sharma M, Radwan J, White R, Diwadkar VA. Structural brain abnormalities and suicidal behavior in borderline personality disorder. J Psychiatr Res. 2012;46:516-25.

44. Yang Y, Raine A. Prefrontal structural and functional brain imaging findings in antisocial, violent, and psychopathic individuals: a meta-analysis. Psychiatry Res. 2009;174:81-8.

45. Rogers JC, De Brito SA. Cortical and subcortical gray matter volume in youths with conduct problems: a meta-analysis. JAMA Psychiatry. 2016;73:64-72.

46. Sebastian CL, De Brito SA, McCrory EJ, Hyde ZH, Lockwood $\mathrm{PL}$, Cecil CA, et al. Grey matter volumes in children with conduct problems and varying levels of callous-unemotional traits. J Abnorm Child Psychol. 2016;44:639-49.

47. Schumann G, Loth E, Banaschewski T, Barbot A, Barker G, Buchel $\mathrm{C}$, et al. The IMAGEN study: reinforcement-related behaviour in normal brain function and psychopathology. Mol Psychiatry. 2010;15:1128-39.

48. Albaugh M, Orr C, Chaarani B, Althoff R, Allgaier N, D'Alberto $\mathrm{N}$, et al. Inattention and reaction time variability are linked to ventromedial prefrontal volume in adolescents. Biol Psychiatry. 2017;82:660-8.

49. Wager TD, Atlas LY. The neuroscience of placebo effects: connecting context, learning and health. Nat Rev Neurosci. 2015;16:403-18.

50. Wager TD, Davidson ML, Hughes BL, Lindquist MA, Ochsner KN. Prefrontal-subcortical pathways mediating successful emotion regulation. Neuron. 2008;59:1037-50.

51. Goodman R. Psychometric properties of the strengths and difficulties questionnaire. J Am Acad Child Adolesc Psychiatry. 2001;40:1337-45.

52. Carroll JM, Maughan B, Goodman R, Meltzer H. Literacy difficulties and psychiatric disorders: evidence for comorbidity. J Child Psychol Psychiatry. 2005;46:524-32.

53. Schilling C, Kuhn S, Paus T, Romanowski A, Banaschewski T, Barbot A, et al. Cortical thickness of superior frontal cortex predicts impulsiveness and perceptual reasoning in adolescence. Mol Psychiatry. 2013;18:624-30.

54. Panizzon MS, Fennema-Notestine C, Eyler LT, Jernigan TL, Prom-Wormley E, Neale M, et al. Distinct genetic influences on cortical surface area and cortical thickness. Cereb Cortex. 2009;19:2728-35.

55. Winkler AM, Kochunov P, Blangero J, Almasy L, Zilles K, Fox PT, et al. Cortical thickness or grey matter volume? The importance of selecting the phenotype for imaging genetics studies. Neuroimage. 2010;53:1135-46.

56. Reuter M, Schmansky NJ, Rosas HD, Fischl B. Within-subject template estimation for unbiased longitudinal image analysis. Neuroimage. 2012;61:1402-18. 
57. Fischl B, van der Kouwe A, Destrieux C, Halgren E, Segonne F, Salat $\mathrm{DH}$, et al. Automatically parcellating the human cerebral cortex. Cereb Cortex. 2004;14:11-22.

58. Fischl B, Sereno MI, Dale AM. Cortical surface-based analysis. II: Inflation, flattening, and a surface-based coordinate system. Neuroimage. 1999;9:195-207.

59. Fischl B, Salat DH, van der Kouwe AJ, Makris N, Segonne F, Quinn BT, et al. Sequence-independent segmentation of magnetic resonance images. Neuroimage. 2004;23(Suppl 1):S69-84.

60. Fischl B, Dale AM. Measuring the thickness of the human cerebral cortex from magnetic resonance images. Proc Natl Acad Sci USA. 2000;97:11050-5.

61. Dale AM, Fischl B, Sereno MI. Cortical surface-based analysis. I. Segmentation and surface reconstruction. Neuroimage. 1999;9:179-94.

62. Desikan RS, Segonne F, Fischl B, Quinn BT, Dickerson BC, Blacker D, et al. An automated labeling system for subdividing the human cerebral cortex on MRI scans into gyral based regions of interest. Neuroimage. 2006;31:968-80.

63. Abe C, Ekman CJ, Sellgren C, Petrovic P, Ingvar M, Landen M. Manic episodes are related to changes in frontal cortex: a longitudinal neuroimaging study of bipolar disorder 1. Brain. 2015;138(Pt 11):3440-8.

64. Dennis M, Francis DJ, Cirino PT, Schachar R, Barnes MA, Fletcher JM. Why IQ is not a covariate in cognitive studies of neurodevelopmental disorders. J Int Neuropsychol Soc. 2009;15:331-43.

65. Logan GD, Cowan WB, Davis KA. On the ability to inhibit simple and choice reaction time responses: a model and a method. J Exp Psychol Hum Percept Perform. 1984;10:276-91.

66. Verbruggen F, Logan GD, Stevens MA. STOP-IT: Windows executable software for the stop-signal paradigm. Behav Res Methods. 2008;40:479-83.

67. Coull JT, Middleton HC, Robbins TW, Sahakian BJ. Contrasting effects of clonidine and diazepam on tests of working memory and planning. Psychopharmacology. 1995;120:311-21.

68. Kempton S, Vance A, Maruff P, Luk E, Costin J, Pantelis C. Executive function and attention deficit hyperactivity disorder: stimulant medication and better executive function performance in children. Psychol Med. 1999;29:527-38.

69. Nymberg C, Banaschewski T, Bokde AL, Buchel C, Conrod P, Flor $\mathrm{H}$, et al. DRD2/ANKK1 polymorphism modulates the effect of ventral striatal activation on working memory performance. Neuropsychopharmacology. 2014;39:2357-65.

70. Kirby KN, Petry NM, Bickel WK. Heroin addicts have higher discount rates for delayed rewards than non-drug-using controls. J Exp Psychol Gen. 1999;128:78-87.
71. Krause-Utz A, Oei NY, Niedtfeld I, Bohus M, Spinhoven P, Schmahl C, et al. Influence of emotional distraction on working memory performance in borderline personality disorder. Psychol Med. 2012;42:2181-92.

72. Prehn K, Schulze L, Rossmann S, Berger C, Vohs K, Fleischer M, et al. Effects of emotional stimuli on working memory processes in male criminal offenders with borderline and antisocial personality disorder. World J Biol Psychiatry. 2013;14:71-8.

73. Egner T, Etkin A, Gale S, Hirsch J. Dissociable neural systems resolve conflict from emotional versus nonemotional distracters. Cereb Cortex. 2008;18:1475-84.

74. Etkin A, Egner T, Peraza DM, Kandel ER, Hirsch J. Resolving emotional conflict: a role for the rostral anterior cingulate cortex in modulating activity in the amygdala. Neuron. 2006;51:871-82.

75. Ochsner KN, Hughes B, Robertson ER, Cooper JC, Gabrieli JD. Neural systems supporting the control of affective and cognitive conflicts. J Cogn Neurosci. 2009;21:1842-55.

76. Rahm C, Liberg B, Wiberg-Kristoffersen M, Aspelin P, Msghina M. Rostro-caudal and dorso-ventral gradients in medial and lateral prefrontal cortex during cognitive control of affective and cognitive interference. Scand J Psychol. 2013;54:66-71.

77. Whalen PJ, Bush G, McNally RJ, Wilhelm S, McInerney SC, Jenike MA, et al. The emotional counting Stroop paradigm: a functional magnetic resonance imaging probe of the anterior cingulate affective division. Biol Psychiatry. 1998;44:1219-28.

78. Petrovic P, Dietrich T, Fransson P, Andersson J, Carlsson K, Ingvar M. Placebo in emotional processing--induced expectations of anxiety relief activate a generalized modulatory network. Neuron. 2005;46:957-69.

79. Schiller D, Delgado MR. Overlapping neural systems mediating extinction, reversal and regulation of fear. Trends Cogn Sci. 2010;14:268-76.

80. Gogtay N, Giedd JN, Lusk L, Hayashi KM, Greenstein D, Vaituzis AC, et al. Dynamic mapping of human cortical development during childhood through early adulthood. Proc Natl Acad Sci USA. 2004;101:8174-9.

81. Yarkoni T, Poldrack RA, Nichols TE, Van Essen DC, Wager TD. Large-scale automated synthesis of human functional neuroimaging data. Nat Methods. 2011;8:665-70.

82. Maier S, Perlov E, Graf E, Dieter E, Sobanski E, Rump M, et al. Discrete global but no focal gray matter volume reductions in unmedicated adult patients with attention-deficit/hyperactivity disorder. Biol Psychiatry. 2016;80:905-15.

83. Reuter M, Tisdall MD, Qureshi A, Buckner RL, van der Kouwe AJ, Fischl B. Head motion during MRI acquisition reduces gray matter volume and thickness estimates. Neuroimage. 2015;107:107-15.

\section{Affiliations}

Frida Bayard ${ }^{1} \cdot$ Charlotte Nymberg Thunell ${ }^{1}$ Christoph Abé ${ }^{1} \cdot$ Rita Almeida $^{2} \cdot$ Tobias Banaschewski $^{3}$. Gareth Barker $\mathbb{D}^{4}$. Arun L. W. Bokde ${ }^{5}$ - Uli Bromberg ${ }^{6}$. Christian Büchel ${ }^{6}$ - Erin Burke Quinlan ${ }^{7}$. Sylvane Desrivières $\mathbb{1}^{7} \cdot$ Herta Flor $^{8,9} \cdot$ Vincent Frouin $^{10} \cdot$ Hugh Garavan $^{11} \cdot$ Penny Gowland ${ }^{12} \cdot$ Andreas Heinz $^{13}$. Bernd Ittermann ${ }^{14}$. Jean-Luc Martinot ${ }^{15}$. Marie-Laure Paillère Martinot ${ }^{16}$. Frauke Nees ${ }^{3,8}$.

Dimitri Papadopoulos Orfanos $\mathbb{1}^{10} \cdot$ Tomáš Paus $^{17} \cdot$ Luise Poustka $^{18,19} \cdot$ Patricia Conrod $^{20} \cdot$ Argyris Stringaris $^{21}$. Maren Struve ${ }^{3} \cdot$ Jani Penttilä ${ }^{22} \cdot$ Viola Kappel $^{23} \cdot$ Yvonne Grimmer $^{3} \cdot$ Tahmine Fadai $^{6} \cdot$ Betteke van Noort $^{24}$. Michael N. Smolka $\mathbb{1}^{25} \cdot$ Nora C. Vetter ${ }^{25} \cdot$ Henrik Walter $\mathbb{B}^{13} \cdot$ Robert Whelan $^{26} \cdot$ Gunter Schumann $^{7}$.

\section{Predrag Petrovic ${ }^{1}$ the IMAGEN Consortium}

1 Department of Clinical Neuroscience, Karolinska Institutet, Stockholm, Sweden

2 Department of Neuroscience, Karolinska Institutet,
Stockholm, Sweden

3 Department of Child and Adolescent Psychiatry and Psychotherapy, Central Institute of Mental Health, Medical 
Faculty Mannheim, Heidelberg University, Mannheim, Germany

4 Centre for Neuroimaging Sciences, Institute of Psychiatry Psychology and Neuroscience, King's College London, London, UK

5 Discipline of Psychiatry, School of Medicine and Trinity College Institute of Neuroscience, Trinity College Dublin, Dublin, Ireland

6 University Medical Centre Hamburg-Eppendorf, House W34, 3. OG, Martinistr. 52, 20246 Hamburg, Germany

7 Centre for Population Neuroscience and Stratified Medicine (PONS) and MRC-SGDP Centre, Institute of Psychiatry, Psychology and Neuroscience, King's College London, London, UK

8 Department of Cognitive and Clinical Neuroscience, Central Institute of Mental Health, Medical Faculty Mannheim, Heidelberg University, Mannheim, Germany

9 Department of Psychology, School of Social Sciences, University of Mannheim, 68131 Mannheim, Germany

10 NeuroSpin, CEA, Université Paris-Saclay, F-91191 Gif-surYvette, France

11 Departments of Psychiatry and Psychology, University of Vermont, Burlington, VT 05405, USA

12 Sir Peter Mansfield Imaging Centre School of Physics and Astronomy, University of Nottingham, University Park, Nottingham, UK

13 Department of Psychiatry and Psychotherapy, Campus Charité Mitte, Charité, Universitätsmedizin Berlin, Charitéplatz 1, Berlin, Germany

14 Physikalisch-Technische Bundesanstalt (PTB), Braunschweig and Berlin, Berlin, Germany

15 Institut National de la Santé et de la Recherche Médicale, INSERM Unit 1000 "Neuroimaging and Psychiatry", University Paris Sud-Paris Saclay, University Paris Descartes; Service
Hospitalier Frédéric Joliot, Orsay; and Maison de Solenn, Paris, France

16 Institut National de la Santé et de la Recherche Médicale, INSERM Unit 1000 "Neuroimaging and Psychiatry", University Paris Sud-Paris Saclay, University Paris Descartes; and AP-HP, Department of Adolescent Psychopathology and Medicine, Maison de Solenn, Cochin Hospital, Paris, France

17 Rotman Research Institute, Baycrest and Departments of Psychology and Psychiatry, University of Toronto, Toronto, ON M6A 2E1, Canada

18 Clinic for Child and Adolescent Psychiatry, Medical University of Vienna, Währinger Gürtel 18-20, 1090 Vienna, Austria

19 Department of Child and Adolescent Psychiatry and Psychotherapy, University Medical Centre Göttingen, vonSiebold-Str. 5, 37075 Göttingen, Germany

20 Department of Psychiatry, Université de Montréal, CHU Ste Justine Hospital, Montréal, QC, Canada

21 National Institute of Mental Health, NIH, Bethesda, MD 20892, USA

22 Department of Social and Health Care, Psychosocial Services Adolescent Outpatient Clinic Kauppakatu 14, Lahti, Finland

23 Department of Child and Adolescent Psychiatry Psychosomatics and Psychotherapy, Campus Charité Mitte, CharitéUniversitätsmedizin Berlin, Charitéplatz 1, Berlin, Germany

24 Department of Child and Adolescent Psychiatry Psychosomatics and Psychotherapy, Campus Charité Mitte, CharitéUniversitätsmedizin Berlin, Charitéplatz 1, Berlin, Germany

25 Department of Psychiatry and Neuroimaging Center, Technische Universität Dresden, Dresden, Germany

26 School of Psychology and Global Brain Health Institute, Trinity College Dublin, Dublin, Ireland 